\title{
Spatial Models Applied on Modern Epidemiological Research: An Example of Malignant Neoplasms of Larynx, Trachea, Bronchus and Lung
}

\author{
Sifaki-Pistolla Dimitra ${ }^{1}$, Pistolla Georgia ${ }^{2}$ \\ ${ }^{1}$ Epidemiology researcher, GISanalyst, Facutly of Medicine, University of Crete, Iraklion, Crete \\ ${ }^{2}$ Mathematician, Facutly of Medicine, University of Crete, Iraklion, Crete
}

Email address:

spdimi11@gmail.com (D. Sifaki-Pistolla),geopist@gmail.com (G. Pistolla)

\section{To cite this article:}

Sifaki-Pistolla Dimitra, Pistolla Georgia. Spatial Models Applied on Modern Epidemiological Research: An Example of Malignant Neoplasms of Larynx, Trachea, Bronchus and Lung. Science Journal of Public Health. Special Issue: Spatial Analysis and Mathematics in Health Research, during Times of Global Socio-Economic Instability. Vol. 3, No. 3-1, 2015, pp. 30-34. doi: 10.11648/j.sjph.s.2015030301.16

\begin{abstract}
Background: The effectiveness of the Greek Health policies is highly doubted, especially in times of economic crisis. We selected a disease associated with one of the highest causes of mortality in Greece to explore this phenomenon. Methods: The number of deaths due to malignant neoplasms of larynx, trachea, bronchus and lung in 2001 and 2006 was used. Mortality rates were analyzed in relation to socioeconomic factors, through Cluster Analysis k-means. Finally, prediction of their variance across the different area of Greece in 2001 and 2006 was fulfilled by the interpolation method of ordinary kriging. Results: Prefectures of the same administrative region are characterised by different behavior while they may match with Prefectures of other administrative regions. In the prediction map, mortality rates range from 0.53 to 1.31 , in 2001 and from 0.66 to 1.27 , in 2006. There is an increase of mortality from one year to another, especially in some Prefectures that move from low clusters in 2001 to very high ones in 2006. Conclusions: This study outlines the regional and spatial inequalities in health, which could be scientifically revealed through the study of health data and their trends. We suggest the promotion of health maps for communication among public health researchers and decision makers.
\end{abstract}

Keywords: Malignant Neoplasms, Mortality Rates, Spatial Models, Kriging-Interpolation, Cluster Analysis

\section{Introduction}

Lung cancer is the leading cause of death in all developed countries, the fourth cause in Europe and third in Greece. Cancer of larynx covers lower ranks in Europe and Greece, whereas in the USA the five-year relative survival estimates are $13 \%$ for men and $17 \%$ for women. From 1975 to 2003 there is a 64.5 adjusted to age-specific incidence rate for both genders and all races [1]. Cancer caused about six million deaths in 1990, 3.4 million of which were observed in men. Approximately 2.4 million cancer deaths occurred in countries with developed market economies and formerly socialist economies in Europe. By 1990, therefore, there were already 50\% more cancer deaths in less developed countries compared to developed countries. Lung cancer, trachea, bronchus, and lung comprise the leading cancer sites of worldwide cancer deaths. Interestingly, lung cancer was already the third leading cause of mortality among women in
1990, accounting for an estimated 237000 deaths (slightly more deaths compared to cervical cancer deaths, at 200000 ) $[2,3]$.

Reliable information on causes of death is essential for the development of national and international health policies for prevention and control of disease and injury. Noncommunicable diseases are, however, already a major public health challenge for all regions [2]. Many sources provide adequate information on the basic population demographic characteristics of each region in the world. Cancer researchers have a long tradition of presenting data in a spatial representation, or through maps, in order to communicate population-based cancer statistics. Historically, health data were being presented in printed atlases where the map producer selected the content and format. The availability of geographic information systems (GIS) with 
comprehensive mapping and spatial analysis capability has vastly expanded the number of producers and consumers of health maps, including policymakers as well as the general public [4-6].

The purpose of this study is not only to produce such maps and promote their use in the communication among public health researchers or politicians, but also to analyse the mortality rates of larynx, lung, bronchus and trachea cancer in Greece, in 2001 and 2006. The present study focuses on both the present distribution of these mortality rates and their clustering, combined with various health determinants per Prefecture [7,8], as well as in its expected future variance through prediction maps. Ii is an environmental study, and in particular, a study of spatial clusters. As it is highlighted in this article, both preventive and treatment measures should be based, in any case, on local political strategies and policies [9]. In addition, cooperation among countries for the adoption of preventive/ treatment measures, in a way that aims at developing a common strategy against cancer, is the final goal at a European level.

\section{Materials and Methods}

\subsection{Data Source}

Our data is retrieved from the Greek National Statistical Authority, at 2001 census [16]. For the present geographical study, we used mortality and socioeconomic data. Mortality data included number of deaths from malignant neoplasms of larynx, trachea, bronchus and lung in 2001 and 2006 per gender and Prefecture of Greece. Socioeconomic data consisted of geographical location (urban, rural, lowland, semi-mountainous, and mountainous), GDP, education level (secondary, postsecondary and other education level), marital status (single, married, or other), and number of clinic beds in particular regions (per Prefecture) [17]. As far as spatial data is concerned, they were collected from the National geographical data organization (geodata.gov), including the coastline of the whole of Greece and its Prefectures, formed in the EGSA 87 (Greek National Georeference datum 87 with projection: Greek Grid) [18 ].

\subsection{Epidemiological and Statistical Analysis}

Firstly, the standardised mortality ratios for each Prefecture of Greece were calculated according to the direct method of standardization [19], stratifying by gender and cause of death. These ratios were grouped according to ICD 10 categories and the grouping of 65 of European Union [20].

Then these mortality rates were combined with the previously mentioned socioeconomic factors through cluster analysis (K-means) in Statistica 8.00, per Prefecture [21,22]. The mortality rates were grouped in three categories for 2001 (low, medium, high), and they refered to a range of numbers from 0.09 to 2.38. As for 2006, there were four categories (low, medium, high and very high) ranging from 0.27 to 2.44 . The number of clusters of each year was defined by the optimum centroids of each cluster.

\subsection{Spatial Analysis}

The results of the statistical analysis were distributed in two maps of spatial clusters at a regional level (figure1). Distribution of both 2001 and 2006 results is presented separately per Prefecture. Furthermore, another set of two maps was created (figure 2) in order to estimate the variation in the distribution of mortality rates in 2001 and 2006 separately, and in order to outline the possible increase or decrease in this distribution. This was applied through Interpolation methods in Gis Arc map (specifically, according to the ordinary kriging - prediction maps) $[10,11]$. Kriging is based on statistical models that include autocorrelation, and refers to the statistical relationships among the measured points. This method is able to produce a prediction surface and also provide some measure of the certainty or accuracy of the predictions [12-15].

\section{Results}

Malignant neoplasms of larynx, trachea, bronchus and lung appear to be of high intensity among most of Greece's Prefectures. If we analyze them in relation with various socioeconomic factors they change the epidemiological impact of the disease in Greece.

The results of the cluster analysis depict a grouping of Prefectures that are of similar behavior in terms of mortality rates and socioeconomic factors. In 2001, most of Greece's Prefectures appear to be of medium or low mortality ratio. The Pr. of Thessaloniki and Attiki are the only exceptions, reaching a higher level for this ratio. We also founnd other groups that originally seemed unlikely. Such examples are Pr. of Lasithi and Rethimno (which are in the island of Crete) with Pr. of Rodopi, Xanthi, Drama and Trikala, which are Pr. of low levels. Similar example, for medium levels is a grouping of Pr. of Chania and Irakleio, with Pr. of Dodekanisos, Evros and Ioannina (figure 1, map to the left). In 2006, the distribution is more heterogeneous. Again, many Prefectures are at the same cluster, irrespective of whether they are of different administrative regions; for instance, Pr. of Rethimno, Fokida and Fthiotida (figure 1- map to the right). At the same time, we find Prefectures that move from low clusters in 2001 into extremely high clusters in 2006. Only five years later, the mortality distribution changes noticeably. On the other hand the Pr. of Thessaloniki and Attiki remain at high levels in both years with a small internal increase (figure 1).

In addition, we estimated and predicted the variance of the phenomenon in 2001 and 2006 separately (figure 2). These maps could give an answer in two research questions: firstly, what is the change of the variance between 2001 and 2006 and secondly, what is the prediction of the spatial variance in 2001 and then in 2006. This refers to a spatial prediction which in our study is applied both at a national and a regional level.

The prediction map of 2001 estimates a range of mortality rates that are between 0.53 to 1.31. Higher rates present in Pr. 
of Attiki and lower rates in Pr. of Evros. If we associate these results with those of figure 1 (map ot the right) of 2006, it is revealed that they have proved to be totally right (as an estimation) and the final distribution is the same. Moreover, what offers a piece of new information is the prediction map of 2006 (figure 2, map to the right), as it proves two basic things: firstly, a national stability or decrease of the mortality rates, and secondly, a regional high increase at some Prefectures. Mortality rates range from 0.66 to 1.27 and one could assume that we should expect a decrease in some areas in 2006. The truth is exactly the opposite. In various Prefectures there would be a huge increase in the mortality rates of malignant neoplasms of larynx and trachea, bronchus and lung. Such are the Prefectures of Karditsa, Aitoloakarnania, Evritania, Grevena, Preveza and Arta. These Prefectures will rise from 0.80 or 1.10 to $0.90-1.29$, in 2006 .

It should be underlined that the following images reflect each step of the process, and prove that the kriging function works effectively that way, and provides correct results. Specifically, it is obvious from the diagram of measured and predicted values, that there is a mathematically satisfying approach among the data. This means that the specific model provides satisfying results.

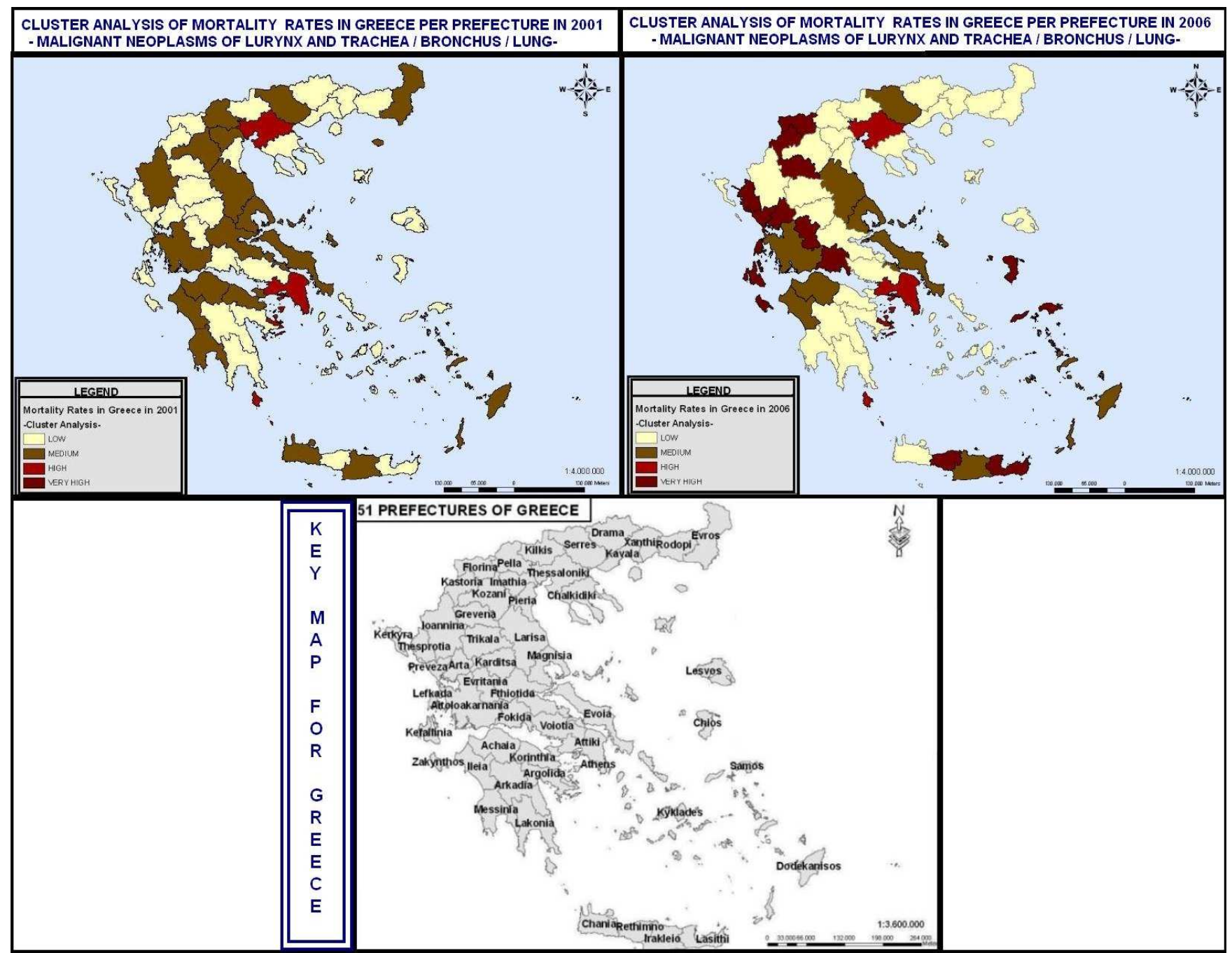

Figure 1. Maps of cluster analysis of mortality rates in Greece in 2001 and 2006, per Prefecture for malignant neoplasms of larynx and trachea, bronchus and lung. 


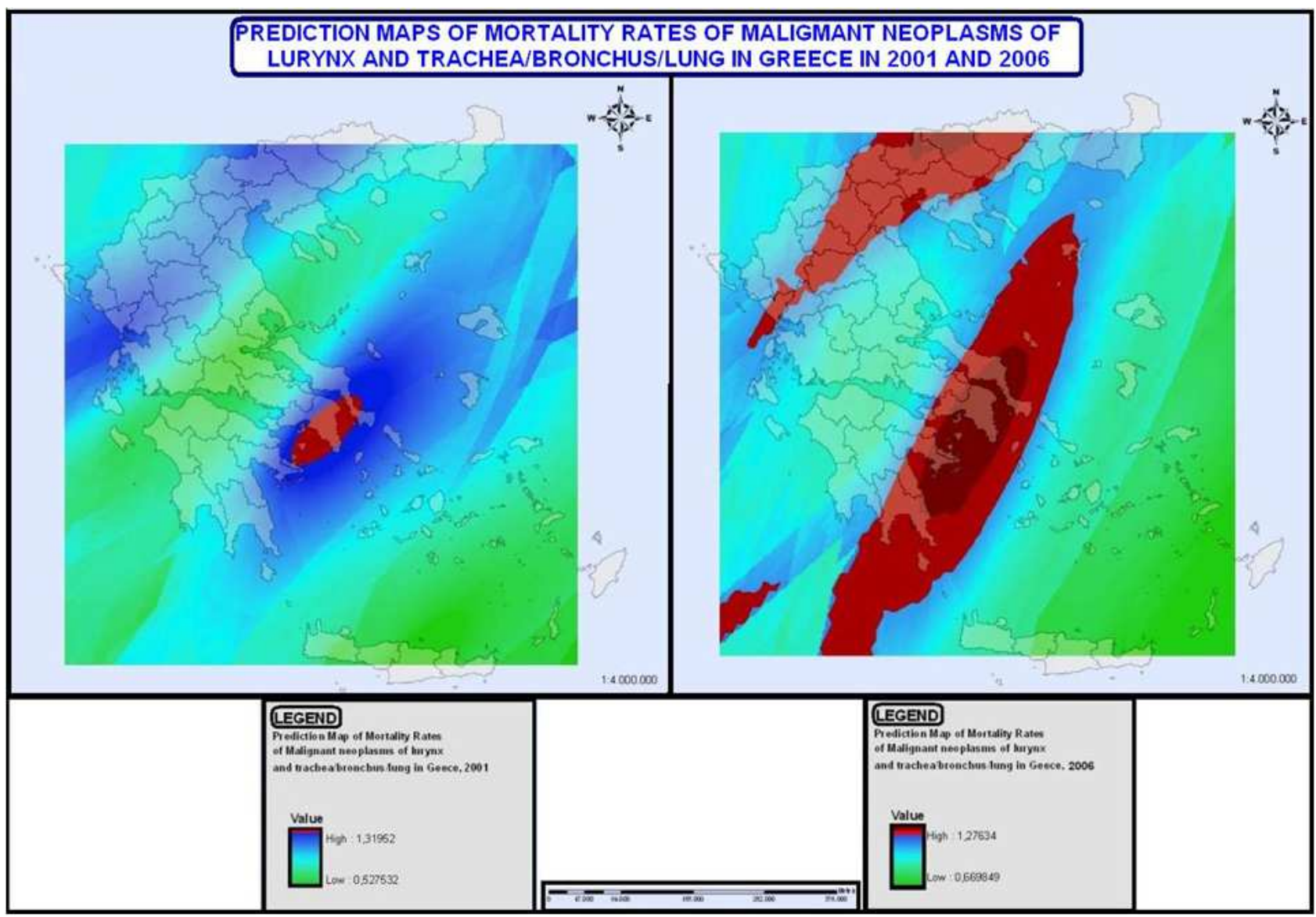

Figure 2. Prediction maps of mortality rates in Greece in 2001 and 2006, per Prefecture for malignant neoplasms of larynx and trachea, bronchus and lung.

\section{Discussion}

Cancer comprises a major worldwide health problem and is considered to be an epidemic phenomenon which constitutes the second major cause of death after cardiovascular diseases. The World Health Organisation (WHO) estimates that 84 million people will die from cancer from 2005 to 2015 with no intervention, while $30-40 \%$ can be treated effectively; which means that proper prevention or treatment measures could help in reducing the incidence of cancer. Cancer mortality rates vary among different countries and their Prefectures or states, by gender and age which outlines the need for accurate epidemiological surveillance.

Regional mortality rates are complex phenomena that are affected by socioeconomic and environmental parameters [23-25]. The association between socioeconomic factors and mortality has been recognized since the Middle Ages. However, health policies have proved to be ineffective in eliminating disparities that contribute to mortality [9]. So, what could possibly be the reason for such policies to not work effectively? As far as Greece is concerned, we have just proved that by taking mortality rates of malignant neoplasms of larynx, trachea, bronchus and lung as a case study, political strategies and measures regarding diseases, should be taken after a specialized extensive epidemiological research at a local level. More specifically, we suggest
Prefectures as a potentially proper research scale, although smaller scales could also be useful.

In modern Greece there is a high risk of deterioration of health services as well as of general levels of public health, especially in times of economic crisis. Even mortality from diseases such as cancer, is likely to be increasing since the whole health care system is unable to offer effective healthoriented services. A basic solution to this problem would be to apply measures customised to local special needs and regional characteristics, taking into consideration both socioeconomic and environmental factors.

\section{Conclusions}

This study revealed several regional and spatial inequalities in health and would be the first step of an extensive study of malignant neoplasms in Greece.

\section{References}

[1] L.A.G. Ries, D. Harkins, M. Krapcho, A. Mariotto, B.A. Miller, E.J. Feuer, L. Clegg, M.P. Eisner, M.J. Horner, N. Howlader, M. Hayat, B.F Hankey and B.K. Edwards, Seer Cancer Statistics Review, 1975-2003. National Cancer Institute. Bethesda, http://seer.cancer.gov/csr/1975_2003/, based on November 2005 SEER data submission, posted to the SEER web site, (2006). 
[2] J.L.M. Christopher and A.D. Lopez, Mortality by cause for eight regions of the world: Global Burden of Disease Study, The Lancet, 349 (1997) 1269 - 1276.

[3] R. Peto, J. Boreham, A.D. Lopez and M.T.C. Heath, Mortality from tobacco in developed countries: indirect estimation from national vital statistics, The Lancet, 339 (1992) 1268-1278.

[4] F.P. Boscoe, M.H. Ward and P. Reynolds, Current practices in spatial analysis of cancer data: data characteristics and data sources for geographic studies of cancer, International Journal of Health Geographics, 3 (2004) 28.

[5] G.M. Jacquez, Current practices in the spatial analysis of cancer: flies in the ointment, International Journal of Health Geographics, 3 (2004) 22.

[6] F. Buntinx, H. Geys, D. Lousbergh, G. Broeders, E. Cloes, D. Dhollander, L. Op De Beeck, J. Vanden Brande, A. Van Waes and G. Molenberghs, Geographical differences in cancer incidence in the Belgian province of Limburg, European Journal of Cancer, 39 (2003) 2060-2065.

[7] E.A. Freda, Viruses, clusters and clustering of childhood leukaemia: a new perspective? European Journal of Cancer, 29 (1993) 1426-1440.

[8] R.Z. Osmar, Principles of Knowledge Discovery in Databases, Data Clustering: Chapter8, Available at: http://www.cs.ualberta.ca/ zaiane/courses/cmput690/slides/Ch apter8/index.html (Accessed: 12 August 2010)

[9] G. Pistolla, P. Prastakos, M. Vassilaki and A. Philalithis, Spatial-Mathematic methods for analysis of indicators of mortality, IJAEST, 2 (2010) 135:14.

[10] S.B. Bell, R.E. Hoskins, L.W. Pickle, and D. Wartenberg, Current practices in spatial analysis of cancer data: mapping health statistics to inform policymakers and the public, International Journal of Health Geographics, 5 (2006) 49.

[11] X. Emery, Simple and Ordinary Kriging Multigaussian Kriging for Estimating recovevearble Reserves, Mathematical Geology, 37 (2005) 295-319.

[12] G.W.A. Heine, Controlled Study of Some Two-Dimensional Interpolation Methods, COGS Computer Contributions, 3 (1986) 60:72.

[13] A.B. McBratney and R. Webster, Choosing Functions for Semi-variograms of Soil Properties and Fitting Them to Sampling Estimates, Journal of Soil Science, 37 (1986) 617:639.
[14] C.A. Pope, R.T. Burnett, M.J. Thun, E.E. Calle, D. Krewski, K. Ito, G.D. Thurston, Lung Cancer, Cardiopulmonary Mortality and Long-term Exposure to Fine Particulate Air Pollution, Journal of American Medical Association, 287 (2002) 11321141 .

[15] M.A. Oliver, Kriging: A Method of Interpolation for Geographical Information Systems, International Journal of Geographic Information Systems, 4 (1990) 313:332.

[16] M. Anderberg, Cluster Analysis for Applications, (1973) New York :Academic Press.N. Krieger, Place, Space, and Health: GIS and Epidemiology, Epidemiology J, 14 (2003) 384-385.

[17] Hellenic Statistical Authority, 2008. Number of deaths. Available at: http://www.statistics.gr/portal/page/portal/ESYE (Accessed: 10/10/2010)

[18] Greek National cadastre and cartography organization, Greek free geographical data,. Available at: http://geodata.gov.gr/geodata/index.php?option=com_sobi2\&c atid=16\&Itemid=10 (Accessed at: 30/4/2011).

[19] M. Pagano and K. Gauvreau, Principles of Biostatistics, Harvard School of Public Health, (1996) Buhbury Press.

[20] European Commission, Eurostat, 2008-2011. E.U Standards for classification. Available at: http://epp.eurostat.ec.europa.eu/portal/page/portal/eurostat/ho me (Accessed: 10 August 2008)

[21] European Commission, Eurostat, 2008-2011. NUTS 2 level. Available at: http://ec.europa.eu/eurostat/ramon/nuts/codelist_en.cfm?list $=$ n uts , (Accessed: 10 August 2008)

[22] K. Bailey, Cluster Analysis, D.Heise Sociological Methodology, (1975) San Francisco : Jossey- Bass.

[23] E. Crocett and P. Carli, Unexpected reduction of mortality rates from melanoma in males living in central Italy, European Journal of Cancer, 39 (2003) 820:821.

[24] P.N. Post, P.J.M. Kil, M.A. Crommelin, R.F.M. Schapers and J.W.W. Coebergh, Trends in incidence and mortality rates for prostate cancer before and after prostate-specific antigen introduction, A registry-based study in Southeastern Netherlands, 1971-1995, European Journal of Cancer, 34 (1998) 707-709.

[25] H. Van, C.S. Victor, A.P. Adam, N. Ronaldd, W.K. Jonathane, K.P. Davida, K. Sungg and D. Joel, Association of Long-term Air Pollution With Ventricular Conduction and Repolarization Abnormalities, Epidemiology J, 22 (2011) 773-780. 